\title{
A DIALLEL CROSS METHOD FOR THE RECOGNITION OF $S$ ALLELE HOMOZYGOTES IN TURNIP, BRASSICA CAMPESTRIS L. SSP. RAPIFERA
}

\author{
G. R. MACKAY \\ Scottish Plant Breeding Station, Pentlandfield, Roslin, Midlothian, Scotland
}

Received 4.x.76

\begin{abstract}
SumMary
A diallel cross method of recognising $S$ allele homozygotes in turnip, Brassica campestris L. ssp. rapifera, is described. By selfing an individual presumed to be heterozygous at its $S$ locus, and raising a progeny of seven plants, there is a high probability $(P=0.99)$ that at least one and possibly both $(P=0.74)$ $S$ homozygotes will be included amongst them. By crossing the inbreds in a full diallel and establishing the compatibility status of each cross, in most cases both $S$ homozygotes will be recognised. In the exceptional case, complete dominance of one allele in both pollen and stigma, the recessive hornozygote only may be recognised $(P=0 \cdot 86)$. It is suggested that this method, in conjunction with U.V. fluorescence microscopy for determining compatibilities, may offer an improvement on previously published methods for this type of material. Results confirm the sporophytic control of the incompatibility in Brassica campestris and the multi-allelic nature of the $S$ locus.
\end{abstract}

\section{INTRODUCTION}

THE turnip, Brassica campestris ssp. rapifera, is an oubtreeder, possessing in common with other self-incompatible crucifers, a sporophytically controlled, multi-allelic incompatibility system (Bateman, 1955; Thompson, 1957; Richards and Thurling, 1973). Plant breeders have successfully exploited this system and the production of Fl hybrids, as proposed by Odland and Noll (1950), is now commonplace. The possibility of using self-incompatibility to produce novel inter-species hybrids is also being investigated (Mackay, 1973).

One of the first steps in such breeding programmes is the isolation of $S$ allele homozygotes in agronomically suitable material. This is complicated by the four types of relationships that can exist between pairs of alleles (fig. 1). Thompson and Howard (1959) suggested methods of recognising $S$ allele homozygotes in kale, $B$. oleracea var. acephala, by pollination of inbreds with tester plants.

Our experience has shown that turnips are far less amenable to inbreeding and maintenance under glasshouse conditions than are kales. They suffer markedly from inbreeding depression, which is expressed as a severe decline in vigour and fertility. Exposure of deleterious recessives causing gross disturbances, such as chlorophyll deficiency and male and female sterility, is quite common following one generation of selfing.

Under experimental conditions, kales (B. oleracea L. var. acephala), because of the ease with which they can be vegetatively propagated, may be treated as biennial perennials. Within the course of a single season their 
flowering period may be greatly extended by removal of senescent flowering shoots to encourage regrowth. Turnips, on the other hand, are more strictly true biennials. Once flowering is initiated, the food reserves in the "bulb" are mobilised; it then degenerates and frequently rots. The flowering period tends to be relatively short and the plants rapidly senesce, providing little opportunity for retesting, by repeating pollinations, and vegetative propagation of parent plants is not practicable.

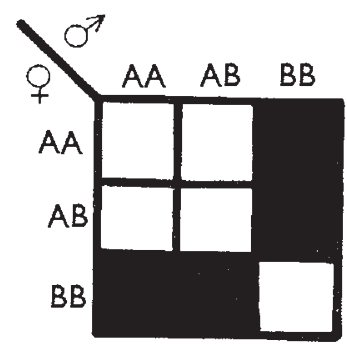

A dominant to $\mathrm{B}$ in pollen and stigma.

TYPE 1

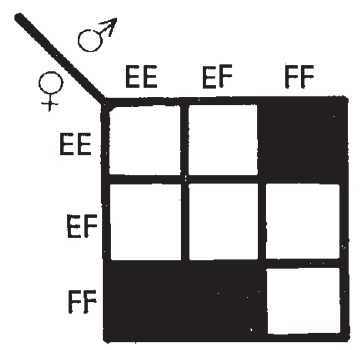

$\mathrm{E}$ dominant to $\mathrm{F}$ in pollen, codominant in stigma.

TYPE 3

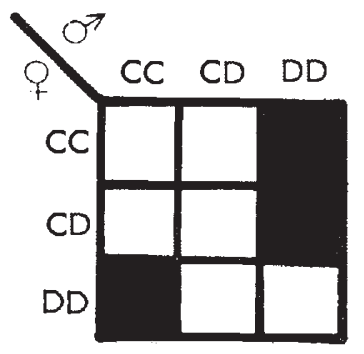

C dominant to $\mathrm{D}$ in stigma, codominant in pollen.

TYPE 2

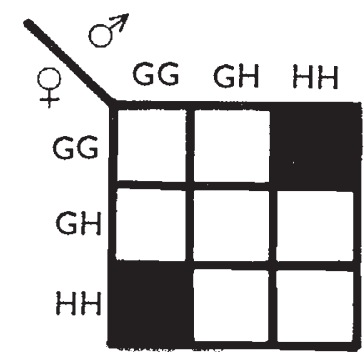

Both alleles, active in pollen and stigma.

TYPE 4

FIG. 1.-Types of dominance relationships between pairs of $S$ alleles. $\square=$ Incompatible cross; $=$ compatible cross.

Consequently, in deciding on a system of $S$ allele homozygote identification, it was felt imperative to use a method that ensured the highest probability of success after one round of crossing. This would then allow isolation of $S$ homozygotes from a wide range of material as quickly as possible, selecting only the more vigorous and fertile inbreds for breeding purposes.

The U.V. fluorescence technique for examination of pollen tube growth (Martin, 1959) is a more reliable technique than seed counting (van Hal and Verhoeven, 1968) and requires fewer pollinations per test-cross to ascertain compatibility relationships. More crosses can be made per complete test, where time, labour and the duration of the flowering period of the plants may be limiting factors. 


\section{MAterials AND METHODS}

Turnip plants, selected from a range of cultivars representing culinary and forage types, were selfed by bud pollination. Samples of the inbred progenies were then test-crossed reciprocally, as open flowers became available, in a series of intra-family diallels. Each member of a diallel was also selfed by bud pollination for seed. All pollinations were made by hand in an insect-proofed glasshouse and inflorescences were covered with "pergamine" bags for 10 days or until removal of pistils, after pollination, as an additional precaution. Pistils of test-crosses were removed and fixed, 24-48 hours after pollination, in $3: 1$ alcohol : acetic acid. After hydrolysis in $1 \mathrm{~N} \mathrm{NaOH}$, overnight at room temperature, they were stained in aqueous soluble aniline blue and examined as squashed preparations using a U.V. microscope, similar to the method of du Crehu (1968). Fixation is not essential, fresh pistils may be hydrolysed and stained for immediate examination, but it has proved convenient to fix and later to exarnine them in batches. The fixative also clears the chlorophyll which can interfere with the stain.

Once compatibility relationships are determined it is usually possible to deduce the $S$ genotypes on inspection (fig. 2, a-d). Progenies derived from selfing recognised $S$ homozygotes were then multiplied and are now maintained by bud pollination between pairs of individuals chosen at random within closed lines.

\section{Results}

Since Spring 1972, 21 intra-family diallels, averaging seven plants per diallel, have been completed. Thirty $S$ allele homozygotes were recognised and 16 are now represented as true breeding, closed lines (table 1).

The 16 extant lines represent the more vigorous and fertile selections, lack of vigour and poor fertility of the rest meant that they were largely self-eliminated from the breeding programme.

In most cases both $S$ homozygotes were isolated from each intra-family diallel (table 1). In one instance, J1Z, where only five inbreds were used. In only two cases, F1Z and S688Z, were all the crosses incompatible.

In diallels $\mathrm{E} 5 \mathrm{Z}, \mathrm{J} 3 \mathrm{Z}$ and $\mathrm{K} 5 \mathrm{Z}$ the results were uninterpretable on any sensible model.

\section{Discussion}

The method of $S$ homozygote recognition, suggested by Thompson and Howard (loc. cit.), initially involves two members of a family produced by selfing a single plant, being test-crossed, one as male the other female, with all other members of that family. Any compatible pollinations permit the partitioning of the inbreds into groups, members of which are then backcrossed to the parent. In turnips the difficulty of maintaining the parent vegetatively precludes this step for routine purposes.

As Thompson and Howard (loc. cit.) pointed out, only compatible pollinations provide the necessary information for the recognition of $S$ homozygotes. The nature of the incompatibility system is such, that in a method 
As Scored

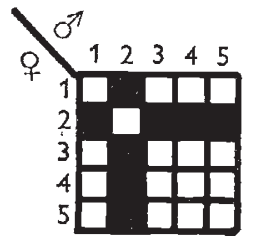

a. Diallel G5Z; Type 1

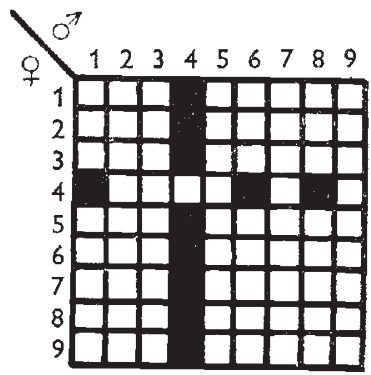

b. Diallel F5Z; Type 2

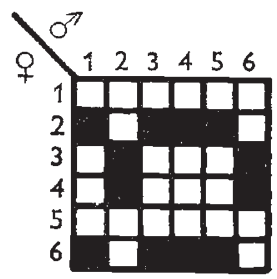

c. Diallel H2Z; Type 3

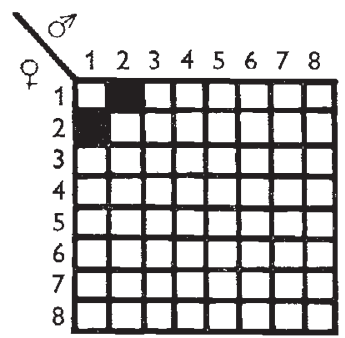

Interpretatiou

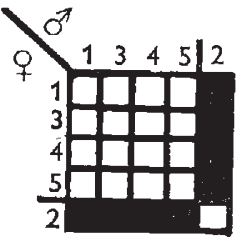

Plant $2=\mathrm{BB}$

Plants $1,3,4$, and 5 either $\mathrm{AA}$ or $\mathrm{AB}$ $\mathrm{A}>\mathrm{B}$ in stigma and pollen

Plants 1,6 and $8=\mathrm{CC}$

Plant $4=$ DD

C $>$ D in stigma

$\mathrm{C}=\mathrm{D}$ in pollen
Plants 3 and $4=\mathbf{E E}$

Plants 2 and $6=\mathrm{FF}$

$\mathrm{E}>\mathrm{F}$ in pollen

$\mathrm{E}=\mathrm{F}$ in stigma

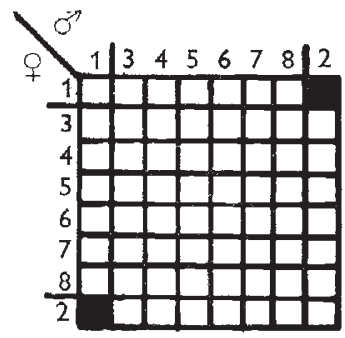

Plant $1=\mathrm{GG}$

Plant $2=\mathrm{HH}$

$\mathrm{G}=\mathrm{H}$ in stigma and pollen

d. Dialle1 E4Z; Type 4

Fig. 2.-Results of four intra-family diallels, illustrating the four types of $S$ allele relationships. $\square=$ Incompatibility; $\square=$ compatible.

using randomly chosen testers, too few compatible crosses is a distinct possibility, requiring additional crosses and/or progeny tests.

In deciding a method to adopt with turnips, it is useful to consider the probabilities of successful isolation of either or both $S$ homozygotes, from a 
TABLE 1

Summary of data from 21 diallels completed over three seasons, 1972, 1973 and 1976

$\begin{array}{lcccc}\begin{array}{c}\text { Diallel } \\ \text { code }\end{array} & \begin{array}{c}\text { Number of } \\ \text { plants }\end{array} & \begin{array}{c}\text { Number of } \\ \text { pollinations }\end{array} & \begin{array}{c}\text { Number of } \\ \text { homozygotes } \\ \text { recognised }\end{array} & \begin{array}{c}\text { Type of } \\ \text { relationship } \\ \text { (fig. 1) }\end{array} \\ \text { A5Z } & 6 & 170 & 2 & 4 \\ \text { E2Z } & 6 & 215 & 2 & 3 \\ \text { E4Z } & 8 & 426 & 2 & 4 \\ \text { E5Z } & 7 & 291 & 0 & \text { see text } \\ \text { F5Z } & 9 & 643 & 2 & 2 \\ \text { F1Z } & 6 & 217 & 0 & \text { see text } \\ \text { G5Z } & 5 & 90 & 1 & 1 \\ \text { H2Z } & 6 & 200 & 2 & 3 \\ \text { I1Z } & 9 & 615 & 2 & 3 \\ \text { I4Z } & 8 & 514 & 2 & 2 \\ \text { J1Z } & 5 & 167 & 2 & \text { see text } \\ \text { J3Z } & 5 & 137 & 0 & 3 \\ \text { J5Z } & 8 & 369 & 2 & \text { see text } \\ \text { K5Z } & 7 & 254 & 0 & 3 \\ \text { L1Z } & 10 & 657 & 2 & 2 \\ \text { L3Z } & 6 & 210 & 2 & 3 \\ \text { M4Z } & 7 & 281 & 1 & \text { see text } \\ \text { N4Z } & 7 & 209 & 2 & 2 \\ \text { S685Z } & 9 & 190 & 2 & \\ \text { S688Z } & 8 & 343 & 0 & \\ \text { S689Z } & 7 & 276 & 2 & \\ \text { Average } & 7 & 308 & & \\ \end{array}$

family derived by selfing a plant presumed to be heterozygous. The expectation that a family of size $k$ contains $r_{1}$ of one genotype, $r_{2}$ of a second, $\ldots r_{j}$ of a $j$ th is

$$
\frac{k !}{r_{1} ! r_{2} ! \ldots r_{j} !} \quad\left(p_{1}\right)^{r_{1}}\left(p_{2}\right)^{r_{2}} \ldots\left(p_{j}\right)^{r_{j}}
$$

where $p_{1}$ is the probability of the first genotype, $p_{2}$ of the second, $\ldots p_{j}$ of the $j$ th (Mather, 1951).

For example, on selfing a heterozygote $S_{1} S_{2}$ one would expect its progeny to segregate in the usual $\frac{1}{4} S_{1} S_{1}: \frac{1}{2} S_{1} S_{2}: \frac{1}{4} S_{2} S_{2}$ Mendelian manner. This being so, given a family of size seven, derived by selfing such a heterozygote, the probability of that family comprising two homozygotes $S_{1} S_{1}$, three heterozygotes $S_{1} S_{2}$ and two homozygotes $S_{2} S_{2}$ is

$$
\frac{7 !}{2 ! 3 ! 2 !}\left(\frac{1}{4}\right)^{2}\left(\frac{1}{2}\right)^{3}\left(\frac{1}{4}\right)^{2}=0 \cdot 1025(P=0 \cdot 10)
$$

Should one wish to calculate the probability of obtaining both homozygotes in a selfed family of size seven, one can find this as one minus (twice the sum of the probabilities of $r_{1}=1,2,3, \ldots 7$ when $r_{3}=0$ plus the probability of obtaining a family in which $r_{1}=r_{3}=0$ and $r_{2}=7$ ). In calculating these probabilities it is necessary to take account of the four types of relationships that can exist between each pair of alleles (fig. 1).

For the purpose of discussion a seven plant diallel, the average of those described, is compared with a test-cross method where two inbreds are crossed, one as male, the other female, with 11 others. The latter is the 
first stage in the method suggestcd for kales (Thompson and Howard, loc. cit.).

It is immediately obvious that choice of testers is critical, since there are six possible combinations of genotypes (table 2). Which "sex" is allocated to which tester also dramatically affects the results of a test involving a type 2 or 3 relationship (fig. 1) and consequently the probability of success.

In a type 1 situation neither a diallel nor crosses to testers will identify both homozygotes, but the latter will be more likely to permit recognition of the recessive homozygote $(\mathbf{P}=0.92)$ than the former $(\mathbf{P}=0.86)$. Since

TABie 2

Possible combinations of genotypes of tester plants, each member of a pair having an equal chance of being used as male or female

\begin{tabular}{|c|c|c|c|c|}
\hline $\begin{array}{l}\text { Frequency: } \\
\text { Genotype: }\end{array}$ & $\begin{array}{l}0.25 \\
A A\end{array}$ & $\begin{array}{c}0 \cdot 50 \\
A a\end{array}$ & $\begin{array}{c}0.25 \\
a a\end{array}$ & Probability \\
\hline $\begin{array}{l}\text { Possible combinations on sampling } \\
2 \text { individuals at random }\end{array}$ & $\begin{array}{l}2 \\
1 \\
1 \\
0 \\
0 \\
0\end{array}$ & $\begin{array}{l}0 \\
1 \\
0 \\
2 \\
1 \\
0\end{array}$ & $\begin{array}{l}0 \\
0 \\
1 \\
0 \\
1 \\
2\end{array}$ & $\begin{array}{l}0 \cdot 0625 \\
0 \cdot 25 \\
0 \cdot 125 \\
0.25 \\
0 \cdot 25 \\
0.0625\end{array}$ \\
\hline
\end{tabular}

the easiest way of identifying the dominant homozygote will be by testcrossing the progeny by selfing the (now) identified recessive homozygote(s) with those of the other inbreds, the test-cross may be slightly more efficient, within the limits of family sizes imposed for this theoretical comparison, providing the type 1 relationship is immediately recognised.

In the casc of types 2 and 3 allele relationships, the diallel may identify both $(\mathbf{P}=0.74)$ or the recessive homozygote only $(\mathrm{P}=0.86)$, but in both instances will distinguish between types 1 and 2 or 3 , because of the reciprocal differences that will be noted (fig. 1). The results of crosses to testers are far less predictable. Given a fortuitous choice of plants, of the correct genotype and "sex", both $S$ homozygotes and their dominance relationship may be recognised $(\mathbf{P}=0.23)$. Failing this, either both $(\mathbf{P}=0 \cdot 17)$, or at least one homozygote $(\mathrm{P}=0.53)$, may be recognised but the results will be indistinguishable from those which might be obtained in a type 1 situation (fig. 3).

With a type 4 relationship the diallel will either recognise both $S$ homozygotes $(\mathbf{P}=0.74)$ or neither $(P=0 \cdot 25)$. The test-cross will identify both $(\mathbf{P}=0 \cdot 11)$ only if each tester is homozygous for a different allele and

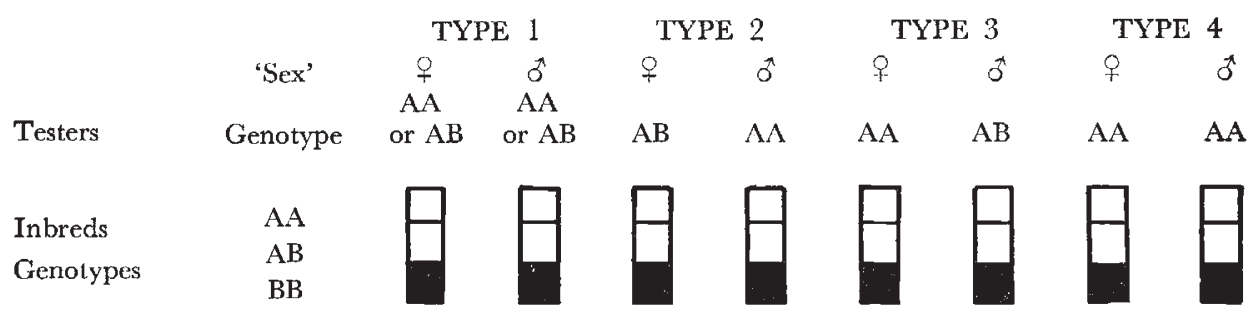

Fig. 3.-Example of test-crosses where the types of $S$ allele relationships are indistinguishable; note, these are not the only instances. $\square=$ Incompatible; $\square=$ compatible. 
neither $(\mathbf{P}=0.29)$ if both are heterozygous. Should one, or other, or both testers be homozygous for one allele there will be some compatible crosses $(\mathbf{P}=0.5)$, but no indication as to which members of these crosses are homozygotes, or what type of $S$ allele relationship exists. Additional crosses will again be necessary to distinguish the results from a type 1 and 2 or 3 (fig. 3).

Obviously modifications of the tester cross method could increase the probability of success. By crossing each tester reciprocally with all other inbreds type 2 and 3 relationships will be differentiated from types 1 and 4, if and when homozygotes are isolated. By increasing the number of testers, the probability that both $S$ homozygotes will be represented amongst them and identified can be improved. However, by increasing the number of inbreds in a diallel, where with the one exception (type 1) the probability of recognising both $S$ homozygotes and their dominance relationship is equal to the probability of their being present, this is also true. A diallel between 11 inbreds would ensure, with the one exception, a high probability of successful recognition of both homozygotes $(\mathbf{P}=0.92)$.

In practice we have averaged $7 \cdot 3$ pollinations per test-cross, more recently reducing this to 4.9 (1976) without loss of information, since it was very seldom necessary to examine more than three pistils to obtain a reliable result. By reducing the number of pollinations to (say) three per cross, an 11 plant diallel would require 330 test pollinations. This should be perfectly feasible, the average reported here being 308 (table 1).

Assuming that the $30 S$ alleles recognised constitute a representative sample and that the four types of relationship are equally prevalent, one may, using the probabilities computed, estimate how many of the 18 interpretable diallels should permit the recognition of 0,1 or $2 S$ allele homozygotes. The frequency of successful recognition of $S$ homozygotes in the work described, does not differ significantly from that expected $\left.\chi_{(2)}^{2}=4 \cdot 8, \quad \mathrm{P}>0.05\right)$. Although, on inspection there seems to be a disproportionately high frequency of type 3 , whether this truly reflects the situation remains to be seen as more $S$ alleles are isolated.

The incompatibility system is subject to modification of expression, by background genotype, environment, age of plant, self-fertility genes and the phenomenon described as "mutual weakening" (van Hal et al., 1968; Nasrallah and Wallace, 1969; Thompson, 1957 and 1972; Richards and Thurling, 1973). It would be expected that such extraneous factors might occasionally interfere with interpretation of the results of any method. Such factors may explain the uninterpretable results of diallels $\mathrm{E} 5 \mathrm{Z}$ and $\mathrm{K} 5 \mathrm{Z}$ (table 1). In the case of E5Z some crosses were, questionably, partially incompatible, having fewer pollen tubes than expected of fully compatible crosses, but too many to assume that they were truly incompatible. With diallel $\mathrm{K} 5 \mathrm{Z}$ one of the putative " heterozygotes " was reciprocally compatible with both " homozygotes", a possible case of pseudo-compatibility.

Poor pollen fertility of some plants of family J3Z confused analysis, although a putative homozygote was identified and a type 2 or 3 situation was indicated, the plants were quite sterile and discarded on these grounds.

Test-crosses between 12 of the $S$ allele homozygous lines has demonstrated, with a single exception, that they are fully cross compatible; thus at least 11 unique $S$ alleles have been isolated so far. The more recently isolated alleles have yet to be tested. Dominance relationships between all possible 
combinations of pairs of alleles is currently being investigated. Results to date, plus the information from the diallels, demonstrates that of 25 pairs, 6 are of type 1, 4 of type 2, 13 of type 3,2 of type 4 (fig. 1).

The work described confirms the sporophytic nature of the $S$ incompatibility system in Brassica campestris and the extremely multi-allelic nature of the $S$ locus. The diallel cross method of $S$ homozygote recognition, discussed and illustrated, seems to offer some advantages over previously described techniques. It offers the added bonus of establishing the dominance relationships between pairs of alleles at the time of recognition, so permitting selection for the dominant ones, which may be more useful for breeding purposes (Ockendon, 1975).

Acknowledgments.-The suggestions and constructive criticism of my colleagues, Dr S. Gowers and Dr I. McNaughton during preparation of this manuscript are gratefully acknow. ledged and thanks are due to Miss Elizabeth Young for her technical assistance.

\section{REFERENCES}

Bateman, A. J. 1955. Self-incompatibility systems in Angiosperms. III. Cruciferae. Heredity, 9, 53-68.

DU CREHU, G. 1968. Early testing of pollen stigma compatibility relationships in Brassica oleracea by fluorescence. Proc. Brassica meeting of Eucarpia, Wellesbourne, Sept. 1968, 34-36.

MACKAY, G. R. 1973. Interspecific hybrids between forage rape (Brassica napus L.) and turnip (Brassica campestric L. ssp. rapifera) as alternatives to forage rape. 1. An exploratory study with single pair crosses. Euphytica, 22, 495-499.

martin, F. w. 1959. Staining and observing pollen tubes in the style by means of fluorescence. Stain Techn., 34, 125-127.

mather, K. 1951. Statistical Analysis in Biology, 4th edition, chap. 2.

NASRAllaH, M. E., AND WALlace, D. H. 1968. The influence of modifier genes on the intensity and stability of self-incompatibility in cabbage. Euphytica, 17, 495-503.

ODLAND, M. L., AND NOLL, C. J. 1950. The utilisation of cross-compatibility and selfincompatibility in the production of Fl hybrid cabbage. Proc. Amer. Soc. Hort. Sci., 55, 391-402.

ockendon, D. J. 1975. Dominance relationships between $\mathrm{S}$ alleles in the stigmas of brussels sprouts (Brassica oleracea var. gemmifera). Euphytica, 24, 165-172.

RICHARDS, R. A., AND THURIING, N. 1973. The genetics of self-incompatibility in Brassica campestris L. ssp. oleifera Metzg. I. Characteristics of S-locus control of self-incompatibility. Genetica, 44, 428-438.

RICHARDS, R. A., AND THURLING, N. 1973. The genetics of self-incompatibility in Brassica campestris L. ssp. oleifera Metzg. II. Genotypic and environmental modification of S locus control. Genetica, 44, 439-453.

THOMPSON, K. F. 1957. Self-incompatibility in natrow-stem kale, Brassica oleracea var. acephala. I. Demonstration of a sporophytic system. J. Genet., 55, 45-60.

THOMPSON, K. F., AND HOWARD, H. w. 1959. Self-incompatibility in marrow-stem kale, Brassica oleracea var. acephala. II. Methods for the recognition in inbred lines of plants homozygous for $\mathrm{S}$ alleles. 7. Genet., 56, 325-340.

THOMPSON, K. F. 1972. Competitive interaction between two $S$ alleles in a sporophyticallycontrolled incompatibility system. Heredity, 28, 1-7.

van hal, J. G., AND VERHoeven, w. 1968. Identification of S alleles in brussels sprouts. Proc. Brassica meeting of Eucarpia, Wellesbourne, Sept. 1968, 32-33.

VAN HAL, J. G., MANGER, A., AND VERHoeven, w. 1968. Estimation of self-incompatibility levels in brusscls sprouts. Proc. Brassica meeting of Eucarpia, Wellesbourne, Sept. 1968, 37-38. 